\title{
DISPONIBILIDADE BIOLÓGICA DO FÓSFORO DE DIFERENTES FONTES PARA EQÜINOS EM CRESCIMENTO'
}

\author{
CARLOS EDUARDO FURTADO², HUGO TOS ${ }^{3}$ e DORINHA MIRIAM SILBER SCHMIDT VITTI ${ }^{4}$
}

\begin{abstract}
RESUMO - Objetivou-se neste estudo, determinar a disponibilidade biológica do P de diferentes fontes, para eqüinos em fase de crescimento. Utilizaram-se dezesseis eqüinos machos em fase de crescimento, submetidos à aplicação de quatro fontes fosfatadas - fosfato de rocha de Tapira (TAP), fosfato de rocha de Patos de Minas (PAT), fosfato bicálcico (BIC) e farinha de osso (FOS) -, adicionadas à dieta basal em quantidades suficientes para fornecer $22 \mathrm{~g}$ de $\mathrm{P} / \mathrm{animal} / \mathrm{dia}$. No $16^{\circ}$ dia, foram-lhes injetados $30 \mathrm{MBq}$ de ${ }^{32} \mathrm{P} / \mathrm{animal}$, e coletaram-se amostras de sangue, fezes e urina, durante sete dias. Foram determinadas as atividades específicas no plasma, fezes e urina e calculou-se a perda endógena fecal e a absorção real de P. Os valores obtidos quanto ao P consumido, $\mathrm{P}$ excretado, $\mathrm{P}$ no plasma e $\mathrm{P}$ retido não apresentaram diferenças estatísticas $(\mathrm{P}>0,05)$. Os valores de absorção real do $\mathrm{P}$ do TAP, PAT, BIC e da FOS foram, respectivamente, 25,23\%, 33,97\%, 31,71\% e 29,36\%. Não houve diferenças estatísticas $(\mathrm{P}>0,05)$ entre as fontes estudadas. Em relação ao BIC, as rochas fosfáticas apresentaram altos valores de disponibilidade biológica.
\end{abstract}

Termos para indexação: cavalos, dieta, necessidades nutricionais, nutrientes minerais, perdas nutricionais, defecação, nutrição animal.

\section{BIOAVAILABILITY OF DIFFERENT SOURCES OF PHOSPHORUS FOR GROWING HORSES}

ABSTRACT - This study aimed at determining the biological phosphorus (P) availability of different $P$ sources for growing horses. Sixteen young male horses submitted to the application of four phosphate sources - rocks phosphate Tapira (RPT) and Patos de Minas (PAT), dicalcium phosphate (DIC) and bone meal (BOM) - added to the basal diet in quantities sufficient to supply $22 \mathrm{~g}$ of $\mathrm{P} / \mathrm{animal} /$ day were used. At the $16^{\text {th }}$ day, the animals were injected with $30 \mathrm{MBq} / \mathrm{animal}$, and blood, feces and urine were collected for seven days. The specific activities of plasma, feces and urine were determined and fecal endogenous loss and true phosphorus absorption were calculated. Values of intake, excreted, plasma and retained phosphorus were unaffected by phosphates sources $(\mathrm{P}>0.05)$. The true absorption values were $25.23 \%, 33.97 \%, 31.71 \%$ and $29.36 \%$ for RPT, PAT, DIC and BOM, respectively. There was no difference $(\mathrm{P}>0.05)$ among sources. Rock phosphate availabilities were high in relation to dicalcium phosphate.

Index terms: horses, diet, nutritional requirements, mineral nutrients, nutritional losses, defecation, animal nutrition.

${ }^{1}$ Aceito para publicação em 23 de novembro de 1999. Extraído da tese de doutorado do primeiro autor. Projeto financiado pela FAPESP.

${ }^{2}$ Zoot., D.Sc., Prof. Associado, Centro de Ciências Agrárias, Dep. de Zootecnia, Universidade Estadual de Maringá (UEM), CEP 87020-900 Maringá, PR. E-mail: cefurtado@uem.br

${ }^{3}$ Eng. Agrôn., D.Sc., Prof. Titular, Dep. de Zootecnia de NãoRuminantes, Fundação Carlos Alberto Vanzolini (FCAV), Universidade Estadual Paulista (UNESP), Caixa Postal 390, CEP 13470-000 Jaboticabal, SP. E-mail: tosi@fcav.unesp.br

${ }^{4}$ Biól., D.Sc., Centro de Energia Nuclear na Agricultura (CENA), Universidade de São Paulo (USP), Caixa Postal 96, CEP 13400-000 Piracicaba, SP. E-mail: vitti@cena.usp.br

\section{INTRODUÇÃO}

Entre os fatores responsáveis pela baixa produtividade do rebanho eqüino nacional, as carências minerais, em especial a de $\mathrm{P}$, ocupam lugar de destaque. As fontes convencionais de $\mathrm{P}$ nas dietas dos cavalos são o fosfato bicálcico e a farinha de ossos, as quais representam cerca de $60 \%$ a $80 \%$ do custo total da mistura mineral.

No Brasil, existe grande interesse pela avaliação do uso de fontes de $\mathrm{P}$ não convencionais. Os fosfatos naturais de rocha despontam como alterna- 
tiva de uso do P em dietas de animais, pois o País possui grandes jazidas de rochas fosfóricas. A partir da década de 80, o uso destas fontes de P vem sendo estudado largamente por inúmeros pesquisadores, principalmente utilizando suínos, aves e ruminantes (Gonzales, 1987; Rostagno et al., 1988; Gomes et al., 1989a,1989b, 1990,1992; Vitti et al., 1989, 1991, 1992a, 1992b; Lopes et al., 1990). A literatura concernente a eqüinos é escassa. Vianna (1990), utilizando fosfato de rocha de Tapira na alimentação de potros em crescimento, concluiu da necessidade de determinar a disponibilidade biológica do $\mathrm{P}$ em fontes não convencionais de $\mathrm{P}$.

Hintz (1990) ressalta que, considerando os eqüinos, a maioria das pesquisas em disponibilidade de nutrientes têm sido conduzidas com outras espécies, e portanto não são válidas para indicar a digestibilidade nesta espécie. Segundo Schryver et al. (1974), Lewis (1985), National Research Council (1989) e Cunha (1991), a disponibilidade biológica do P do fosfato bicálcico, da farinha de osso e do fosfato monossódico varia de $44 \%$ a $47 \%$, podendo ser influenciada pela idade do animal, pela fonte de $\mathrm{P}$ e pela concentração do elemento na dieta.

Este trabalho teve como objetivo determinar a disponibilidade biológica (absorção real) do P de diferentes fontes em dietas para eqüinos em crescimento.

\section{MATERIAL E MÉTODOS}

O experimento foi conduzido no setor de Eqüinocultura da FACV-UNESP, Jaboticabal, SP, e no Setor de Ciências Animais do CENA-USP, Piracicaba, SP. Utilizaram-se 16 eqüinos machos da raça Brasileiro de Hipismo, com idade média de dez meses, e com 257,31 $\pm 21,9 \mathrm{~kg}$. Foram avaliadas quatro fontes fosfatadas oferecidas em quantidades suficientes para fornecer $22 \mathrm{~g}$ de $\mathrm{P} / \mathrm{animal} / \mathrm{dia}$. As fontes (tratamentos) utilizadas foram o fosfato de rocha de Tapira (TAP), fosfato de rocha de Patos de Minas (PAT), fosfato bicálcico (BIC) e farinha de osso (FOS), incorporadas a uma dieta basal sem suplementação de $\mathrm{P}(0,15 \%$ de $\mathrm{P}$ total) (Tabelas 1,2 e 3). As dietas foram oferecidas na forma farelada, e a ingestão de matéria seca $(4,6 \mathrm{~kg} /$ cabeça/dia) foi previamente estabelecida para atender às necessidades de ingestão de P. Após vermifugações e pesagem, os animais foram colocados em boxes individuais e submetidos a um período experimental-I com duração de quinze dias, onde receberam as dietas experimentais para adaptação. No período experimental-II, com duração de sete dias, os animais permaneceram alojados em gaiolas de metabolismo, receberam por injeção via jugular $30 \mathrm{MBq}$ de ${ }^{32} \mathrm{P}$ $\left(\mathrm{Na}_{2} \mathrm{HPO}_{4}\right)$ e coletaram-se amostras de sangue, urina e fezes. Foram determinados os níveis de $\mathrm{P}$ inorgânico no plasma e nas fezes (Fiske \& Subbarrow, 1925), como também a atividade radioativa do plasma, fezes e urina, através do efeito Cerenkov (International Atomic Energy Agency, 1979). Foram determinadas as atividades especí-

TABELA 1. Composição porcentual da dieta basal.

\begin{tabular}{lc}
\hline Ingredientes & Porcentagem na dieta \\
\hline Feno de gramínea moído & 47 \\
Rolão de milho & 48 \\
Farelo de soja $^{1}$ & 1 \\
Núcleo mineral $^{1}$ & 4 \\
\hline Total & 100 \\
\hline Matéria seca (\%) & 95,85 \\
Proteína bruta (\%) & 9,51 \\
Cinzas (\%) & 3,19 \\
Fibra bruta (\%) & 20,26 \\
Extrato etéreo (\%) & 2,79 \\
Energia digestível (kcal/kg) & 2.300 \\
Fósforo (\%) & 0,15 \\
Cálcio (\%) & 0,20 \\
\hline
\end{tabular}

${ }^{1}$ Sem suplementação de fósforo. 2 Valor estimado.

TABELA 2. Composição porcentual dos núcleos minerais.

\begin{tabular}{lcccc}
\hline Ingredientes & Tapira & $\begin{array}{c}\text { Patos } \\
\text { de Minas }\end{array}$ & Bicálcio & $\begin{array}{r}\text { Farinha } \\
\text { de osso }\end{array}$ \\
\hline Fosfato de Tapira & 56,40 & - & - & - \\
Fosfato P. de Minas & - & 74,50 & - & - \\
Fosfato bicálcico & - & - & 38,00 & - \\
Farinha de osso & - & - & - & 65,00 \\
Calcário & - & - & 20,50 & 3,40 \\
Sal comum & 17,50 & 17,50 & 17,50 & 17,50 \\
Caulim & 19,33 & 1,23 & 17,33 & 7,33 \\
MgO & 5,10 & 5,10 & 5,00 & 5,10 \\
Micronutrientes ${ }^{1}$ & 1,67 & 1,67 & 1,67 & 1,67 \\
\hline Total & 100,00 & 100,00 & 100,00 & 100,00 \\
\hline Matéria seca (\%) & 98,03 & 99,39 & 99,55 & 99,74 \\
Cinzas (\%) & 77,73 & 90,63 & 96,27 & 94,25 \\
Fósforo (\%) & 8,20 & 7,83 & 7,72 & 8,75 \\
Cálcio (\%) & 17,02 & 14,15 & 14,73 & 16,94 \\
\hline
\end{tabular}

${ }^{1}$ Composição dos micronutrientes por $\mathrm{kg}$ do produto: $1.500 \mathrm{mg}$ de $\mathrm{Zn}$; $250 \mathrm{mg}$ de $\mathrm{Cu} ; 1.000 \mathrm{mg}$ de $\mathrm{Mn} ; 1.000 \mathrm{mg}$ de Fe; $12,4 \mathrm{mg}$ de Co; $20 \mathrm{mg}$ de I; $2,25 \mathrm{mg}$ de Se e $0,72 \mathrm{~g}$ de $\mathrm{F}$. 
ficas nas fezes e no plasma como porcentagem da atividade injetada, a proporção de $\mathrm{P}$ endógeno, e, conseqüentemente, a disponibilidade biológica ou absorção real (Lofgreen \& Kleiber, 1953). O delineamento experimental foi o de blocos casualizados com quatro repetições e a análise de variância foi realizada utilizando o Statistical Analysis System (SAS Institute, 1986). A comparação de médias foi feita pelo teste de Tukey $(\mathrm{P}<0,05)$.

\section{RESULTADOS E DISCUSSÃO}

Os resultados obtidos quanto aos parâmetros relacionados ao metabolismo do P estão apresenta-

TABELA 3. Análise bromatológica das rações experimentais contendo diferentes fontes de $P$ para eqüinos em crescimento.

\begin{tabular}{lrrrrr}
\hline Composição $^{1}$ & Feno & $\begin{array}{r}\text { Conc. } \\
\text { TAP }^{2}\end{array}$ & $\begin{array}{r}\text { Conc. } \\
\text { PAT }^{2}\end{array}$ & $\begin{array}{r}\text { Conc. } \\
\text { BIC }^{2}\end{array}$ & $\begin{array}{r}\text { Conc. } \\
\text { FOS }^{2}\end{array}$ \\
\hline Matéria seca (\%) & 95,30 & 95,80 & 95,80 & 95,67 & 95,35 \\
Proteína bruta (\%) & 6,34 & 9,20 & 9,80 & 9,63 & 11,47 \\
Cinzas (\%) & 4,61 & 8,41 & 8,77 & 9,21 & 8,90 \\
Fibra bruta (\%) & 34,72 & 11,08 & 11,06 & 10,79 & 10,35 \\
Extrato etéreo (\%) & 1,97 & 3,34 & 3,11 & 3,06 & 3,03 \\
Fósforo (\%) & 0,11 & 0,90 & 1,02 & 0,96 & 1,03 \\
Cálcio (\%) & 0,43 & 1,55 & 1,32 & 1,10 & 1,39 \\
\hline
\end{tabular}

${ }^{1}$ Em $100 \%$ de matéria seca.

2 O fosfato de rocha de Tapira continha $12,80 \%$ de P, $22,69 \%$ de Ca e 1,16\% de F; o fosfato de rocha de Patos de Minas continha 9,70\% de P, 21,93\% de Ca e 1,74\% de F; o fosfato bicálcico continha 17,66\% de P, 23,78\% de $\mathrm{Ca}$ e $0,08 \%$ de F; a farinha de osso continha $11,11 \%$ de P, $21,19 \%$ de Ca e $0,02 \%$ de $\mathrm{F}$. dos na Tabela 4. Considerando os valores do P consumido e do $\mathrm{P}$ no plasma, não houve diferenças $(\mathrm{P}>0,05)$ entre os tratamentos, e estes foram considerados adequados, conforme Teeter et al. (1967), National Research Council (1989) e Ott (1995).

Os níveis de $\mathrm{P}$ excretado na urina, no presente trabalho, se mostraram baixos, correspondendo a aproximadamente $1,5 \%$ do $\mathrm{P}$ consumido. Schryver et al. (1971), fornecendo a pôneis consumo de $\mathrm{P}$ da ordem de $108 \mathrm{mg} / \mathrm{kg} \mathrm{PV}$, obtiveram valores de excreção renal de $19 \mathrm{mg} / \mathrm{kg} \mathrm{PV}$, correspondendo a $17,5 \%$ do $\mathrm{P}$ consumido. Outros trabalhos relataram, entretanto, que eqüinos submetidos a níveis mais baixos de $\mathrm{P}$ consumido (30 a $60 \mathrm{mg} / \mathrm{kg}$ PV), a excreção renal de $\mathrm{P}$ correspondeu a $3 \%$ do $\mathrm{P}$ consumido, sendo que em alguns casos não houve detecção de P na urina. Meyer \& Stadermann (1990) determinaram, em eqüinos, correlação muito baixa entre $\mathrm{P}$ excretado na urina e $\mathrm{P}$ consumido.

Com relação aos valores de $\mathrm{P}$ total excretado nas fezes, não houve efeito $(\mathrm{P}>0,05)$ de tratamento, porém observa-se, no que se refere a PAT, que a excreção do elemento foi menor. $\mathrm{O} P$ excretado nas fezes correspondeu, em média, a 79,8\% do P consumido, demonstrando que a maior parte da eliminação do $\mathrm{P}$ é feita através das fezes, principalmente quando o animal não consome altos níveis do elemento na dieta, o que está de acordo com Schryver et al. (1971), Hintz (1979) e Cymbaluk \& Christensen (1986). Estudos de Hintz \& Schryver (1972), Hintz et al. (1973) e Cymbaluk et al. (1989), utilizando dietas basais suplementadas por fosfato bicálcico, farinha de osso

TABELA4. Parâmetros relacionados ao metabolismo de $\mathbf{P}$ de dietas contendo diferentes fontes de $\mathbf{P}^{1}$ para eqüinos em crescimento ${ }^{2}$.

\begin{tabular}{lccccc}
\hline Parâmetro & TAP & PAT & BIC & FOS & C.V. (\%) \\
\hline P consumido (mg/kg PV) & $89,35 \pm 18,27$ & $96,10 \pm 18,11$ & $105,31 \pm 29,39$ & $100,89 \pm 20,00$ & 9,63 \\
P plasma (mg/100 mL) & $4,69 \pm 0,54$ & $5,46 \pm 0,49$ & $4,93 \pm 0,40$ & $4,89 \pm 0,40$ & 10,91 \\
P urina (mg/kg PV) & $0,11 \pm 0,12$ & $2,64 \pm 5,00$ & $0,34 \pm 0,46$ & $2,76 \pm 4,74$ & 183,54 \\
P fezes (mg/kg PV) & $76,57 \pm 13,66$ & $72,14 \pm 17,55$ & $82,96 \pm 25,04$ & $81,02 \pm 11,18$ & 16,29 \\
P endógeno (mg/kg PV) & $9,63 \pm 2,92$ & $8,13 \pm 2,29$ & $11,26 \pm 5,43$ & $9,65 \pm 3,64$ & 33,42 \\
P absorvido (mg/kg PV) & $22,41 \pm 5,43 b$ & $32,10 \pm 5,15 \mathrm{a}$ & $32,71 \pm 6,45 \mathrm{a}$ & $29,72 \pm 7,28 \mathrm{ab}$ & 14,73 \\
Disponib. biológica (\%) & $25,23 \pm 4,35$ & $33,97 \pm 5,83$ & $31,71 \pm 4,11$ & $29,36 \pm 4,87$ & 16,42 \\
P retido (mg/kg PV) & $12,67 \pm 8,39$ & $21,33 \pm 8,12$ & $22,00 \pm 12,65$ & $17,12 \pm 12,01$ & 38,50 \\
Meia-vida biológica (horas) & $165,43 \pm 88,25$ & $153,57 \pm 94,88$ & $140,05 \pm 69,93$ & $147,42 \pm 36,49$ & 40,74 \\
Peso vivo (kg) & $261,25 \pm 8,5$ & $259,00 \pm 18,00$ & $256,00 \pm 39,09$ & $256,00 \pm 21.44$ & 7,19 \\
\hline
\end{tabular}

1 TAP: fosfato de rocha de Tapira; PAT: fosfato de rocha de Patos de Minas; BIC fosfato bicálcico; FOS: farinha de osso.

2 Valores seguidos de letras iguais nas linhas não diferem entre si pelo teste de Tukey $(\mathrm{P}>0,05)$. 
e fosfato monossódico, obtiveram valores médios de $\mathrm{P}$ excretado nas fezes/P consumido $(76,2 \%)$ próximos ao obtido neste trabalho.

A perda endógena fecal média de $\mathrm{P}$ representou cerca de $12,5 \%$ do $P$ total excretado nas fezes, e quanto ao BIC, o valor correspondeu a $14,0 \%$, o que sugere tendência de melhor absorção de $\mathrm{P}$ neste tratamento. Os valores obtidos de excreção fecal metabólica de P são semelhantes aos relatados por Schryver et al. (1971) e Cymbaluk et al. (1989), os quais obtiveram valores de P endógeno fecal de 11,0 e $17,9 \mathrm{mg} / \mathrm{kg}$ PV, respectivamente.

Considerando a absorção de $\mathrm{P}$, houve diferença $(\mathrm{P}<0,05)$ entre as fontes de $\mathrm{P}$ estudadas, onde TAP apresentou menor valor de $\mathrm{P}$ absorvido, provavelmente em função do menor consumo de $\mathrm{P}$ neste tratamento.

Não houve diferença $(\mathrm{P}>0,05)$ entre as fontes de $\mathrm{P}$ estudadas quanto ao parâmetro disponibilidade biológica (absorção real), cujos valores foram $25,23 \% ; 33,97 \% ; 31,71 \%$ e $29,36 \%$, respectivamente, no que diz respeito a TAP, PAT, BIC e FOS, embora os valores obtidos com PAT e BIC tenham-se mostrado superiores. Comparativamente ao BIC, os valores de absorção real obtidos com TAP, PAT e FOS podem ser considerados altos: $79,6 \% ; 107,1 \%$ e $92,53 \%$, respectivamente. Os valores de disponibilidade biológica mostraram-se próximos das estimativas relatadas pelo National Research Council (1989), e em outros estudos, como os de Schryver et al. (1974), Lewis (1985) e Cunha (1991). Os valores com TAP e PAT foram semelhantes, ou, em alguns casos, superiores, aos obtidos em outras espécies (aves, suínos, coelhos e ruminantes) por Gonzales (1987), Rostagno et al. (1988), Vitti et al. (1989, 1991, 1992a, 1992b), Lopes et al. (1990) e Furlan (1994).

Os valores de P retido não apresentaram diferenças $(\mathrm{P}>0,05)$ entre as fontes de $\mathrm{P}$ estudadas, porém houve tendência de os valores de PAT e BIC se mostrarem superiores. Os dados obtidos em todos os tratamentos indicaram que os animais permaneceram em balanço positivo de $P$.

Considerando-se as curvas de atividade específica (Fig. 1), não houve diferença $(\mathrm{P}>0,05)$ entre os tratamentos. Portanto, não houve diferença no metabolismo do P entre TAP, PAT, BIC e FOS, ou seja, no tempo gasto para que o elemento fosse distribuído aos tecidos e utilizado pelo organismo. Houve tendência de os fosfatos naturais de rocha apresen- tarem tempo mais lento para serem metabolizados, fato, este, talvez, relacionado à forma química do composto e também à sua solubilidade.

A Fig. 2 ilustra os parâmetros relacionados ao
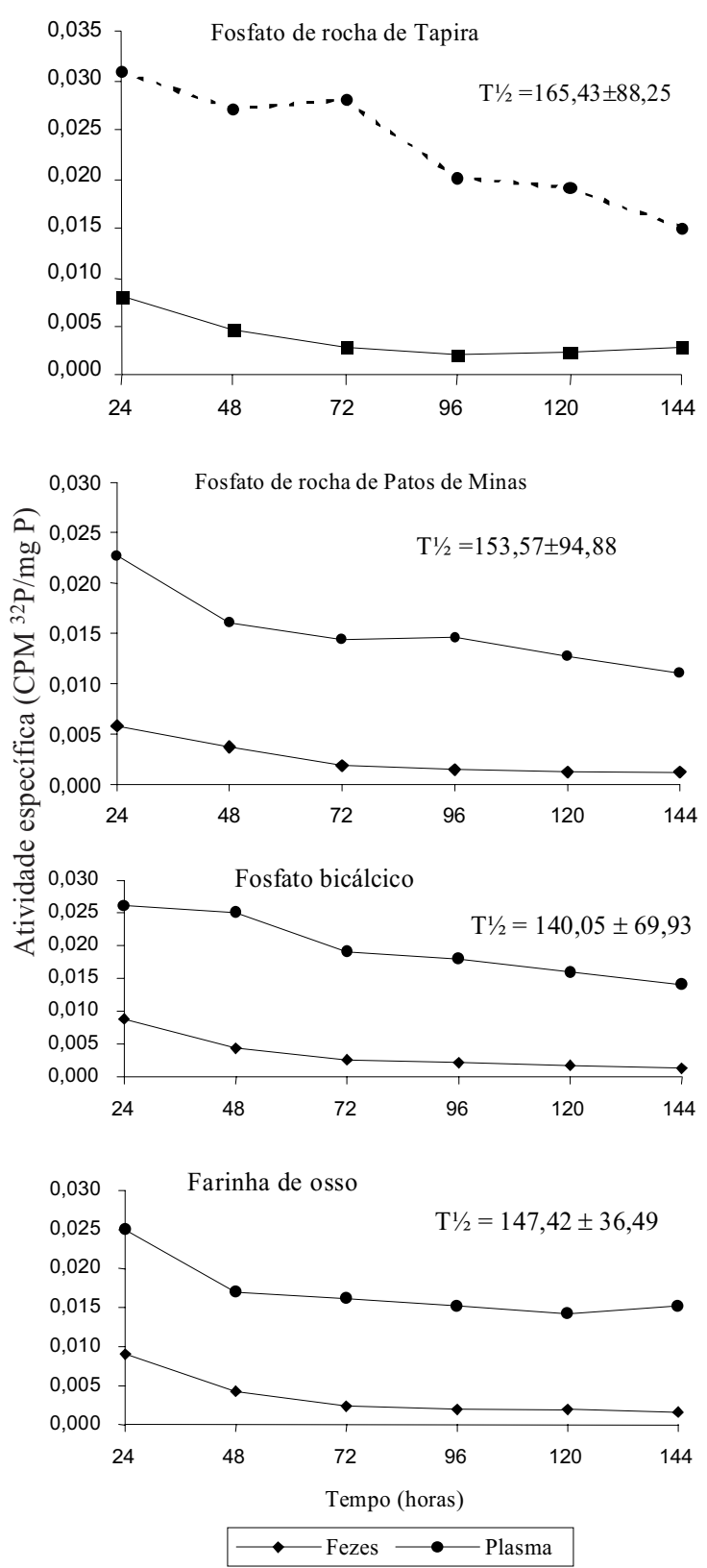

FIG. 1. Curvas de atividades específicas nas fezes e no plasma relativas aos diferentes tratamentos. 


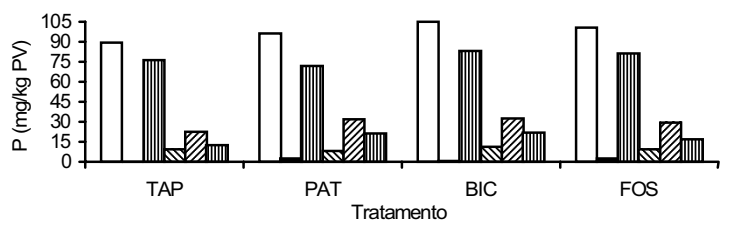

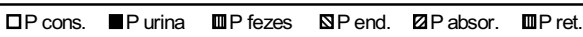

FIG. 2. Distribuição do $P$ das fontes fosfato de rocha de Tapira (TAP), fosfato de rocha de Patos de Minas (PAT), fosfato bicálcico (BIC) e farinha de osso (FOS).

metabolismo de $\mathrm{P}$, permitindo melhor visualização do comportamento do elemento no organismo animal. Observou-se que os tratamentos PAT e BIC apresentaram maiores valores de absorção e retenção de $\mathrm{P}$, refletindo em maiores valores de disponibilidade biológica do elemento.

\section{CONCLUSÕES}

1. Os valores de biodisponibilidade (absorção real) do $\mathrm{P}$ do fosfato de rocha de Tapira, fosfato de rocha de Patos de Minas, fosfato bicálcico e farinha de osso indicam que estas fontes são adequadas aos eqüinos.

2. Considerando os valores de P endógeno fecal, $\mathrm{P}$ absorvido, absorção real, e $\mathrm{P}$ retido, o fosfato de rocha de Patos de Minas e o fosfato bicálcico são as fontes mais eficientes de $\mathrm{P}$ para eqüinos.

3. Relativamente ao fosfato bicálcico, os fosfatos de rocha (de Tapira e de Patos de Minas) apresentam altos valores de biodisponibilidade do elemento, e portanto podem ser utilizados como suplemento de $\mathrm{P}$ em dietas para eqüinos.

\section{REFERÊNCIAS}

CUNHA, T.J. Horse feeding and nutrition. 2.ed. San Diego : Academic, 1991. 445p.

CYMBALUK, N.F.; CHRISTENSEN, D.A. Nutrient utilization of pelleted and unpelleted forages by ponies. Canadian Journal of Animal Science, Ottawa, v.66, n.2, p.237-244, 1986.
CYMBALUK, N.F.; CHRISTISON, G.I.; LEACH, D.H Nutrient utilization by limit and ad libitum fed growing horses. Journal of Animal Science, Champaign, v.67, n.2, p.414-425, 1989.

FISKE, C.H.; SUBBARROW, Y. The calorimetric determination of phosphorus. Journal of Biological Chemistry, Bethesda, , v.66, n.2, p.375-400, 1925.

FURLAN, A.C. Exigência nutricional de fósforo e uso de fosfatos naturais de rocha na alimentação de coelhos em crescimento. Viçosa : Universidade Federal, 1994. 160p. Tese de Doutorado.

GOMES, P.C.; GOMES, M.F.M.; LIMA, G.J.M.M.; FERREIRA, A.S.; BARIONI, W. Disponibilidade de fósforo em alguns fosfatos para suínos. Revista Brasileira de Zootecnia, Viçosa, v.19, n.4, p.306313, 1990 .

GOMES, P.C.; LIMA, G.J.M.M.; BARBOSA, H.P.; GOMES, M.F.M.; BELLAVER, C. Disponibilidade de fósforo nos fosfatos de tapira e fosforindus e na farinha de ossos para suínos. Revista Brasileira de Zootecnia, Viçosa, v.21, n.1, p.83-89, 1992.

GOMES, P.C.; ROSTAGNO, H.S.; COSTA, P.M.A.; PEREIRA, J.A.A.; LIMA, J.A.L. Digestibilidade aparente e verdadeira do fósforo de cinco alimentos determinada em suínos com diferentes idades. Revista Brasileira de Zootecnia, Viçosa, v.18, n.1, p.77-90, 1989a.

GOMES, P.C.; ROSTAGNO, H.S.; COSTA, P.M.A.; PEREIRA, J.A.A.; LIMA, J.A.L. Digestibilidade aparente e verdadeira do fósforo de três fosfatos, determinada em suínos de $13 \mathrm{~kg}$ de peso vivo. Revista Brasileira de Zootecnia, Viçosa, v.18, n.1, p.54-63, 1989b.

GONZALES, C.I.L. Biodisponibilidade de fósforo de seis fontes de fósforo utilizados na alimentação animal. Belo Horizonte : UFMG-Escola de Veterinária, 1987. 98p. Dissertação de Mestrado.

HINTZ, H.F. Factors affecting nutrient availability in the horse. In: GEORGIA NUTRITION CONFERENCE FOR THE FEED INDUSTRY, 1990, Atlanta. Proceedings. Atlanta : [s.n.], 1990. p.181-193.

HINTZ, H.F. Nutrición del caballo. In: EVANS, J.W.; BORTON, A.; HINTZ, H.F.; VLECK, D. van. El caballo. Zaragoza : Acribia, 1979. p.233-275.

HINTZ, H.F.; SCHRYVER, H.F. Availability to ponies of calcium and phosphorus from various supplements. Journal of Animal Science, Champaign, n.34, n.6, p.979-980, 1972.

Pesq. agropec. bras., Brasília, v.35, n.5, p.1011-1016, maio 2000 
HINTZ, H.F.; WILLIAMS, A.J.; ROGOFF, J.; SCHRYVER, H.F. Availability of phosphorus in wheat brain when fed to ponies. Journal of Animal Science, Champaign, v.36, n.3, p.522-525, 1973

INTERNATIONAL ATOMIC ENERGY AGENCY (Vienna, Austria). Laboratory training manual on the use of nuclear techniques in animal research. Vienna, 1979. 300p.

LEWIS, R.L. Alimentação e cuidados do cavalo. São Paulo : Roca, 1985. 365p

LOFGREEN, G.P.; KLEIBER, M. The availability of the phosphorus in alfalfa hay. Journal of Animal Science, Champaign, v.12, n.2, p.366-371, 1953.

LOPES, H.O.S.; VITTI, D.M.S.S.; PEREIRA, E.A.; ABDALLA, A.L.; MORAES, E.A.; SILVA FILHO, J.C.; FICHTER, S.S. Disponibilidade biológica do fósforo de fosfatos naturais para bovinos pela técnica de diluição isotópica. Pesquisa Agropecuária Brasileira, Brasília, v.25, n.3, p.421-425, mar. 1990

MEYER, H.; STADERMANN, B. Use of urine analysis to estimate mineral supply of horses. Advances in Animal Physiology and Animal Nutrition, Hamburg, n.21, p.86-97, 1990.

NATIONAL RESEARCH COUNCIL. Board on Agriculture and Natural Resources. Committee on Animal Nutrition (Washington, Estados Unidos) Nutrient requirements of horses. 5.rev.ed. Washington : National Academy of Sciences, 1989. 112p. (Nutrient Requirements of Domestic Animals).

OTT, E.A. Dietary nutrient allowances for horses. Feedstuffs, Minnetonka, v.64, n.29, p.77-80, 1995

ROSTAGNO, H.S.; SAKOMURA, N.; GOMES, P.C.; SOARES, P.R.; KUANA, S. Exigência nutricional de fósforo e sua disponibilidade em fosfato de rocha e fosfato parcialmente defluorizado para pintos de corte. Revista Brasileira de Zootecnia, Viçosa, v.17, n.3, p.249-257, 1988

SAS INSTITUTE (Cary, Estados Unidos). Statistical analysis system: SAS system for linear models. Cary, 1986. 211p.
SCHRYVER, H.F.; HINTZ, H.F.; GRAIG, P.H Phosphorus metabolism in ponies fed varying levels of phosphorus. Journal of Nutrition, Bethesda, v.101, n.19, p.1257-1264, 1971.

SCHRYVER, H.F.; HINTZ, H.F.; LOWE, J.E. Calcium and phosphorus in the nutrition of the horse. Cornell Veterinarian, Ithaca, v.64, n.4, p.493-515, 1974.

TEETER, S.M.; STILLIONS, M.C.; NELSON, W.E. Maintenance levels of calcium and phosphorus in horses. American Veterinary Medical Association Journal, Schaumburg, v.151, n.12, p.1625-1628, 1967.

VIANNA, D.V.M. Avaliação do uso do fosfato de rocha com baixo teor de flúor na alimentação de eqüinos em crescimento. Jaboticabal : UNESPFCAVJ, 1990. 89p. Dissertação de Mestrado.

VITTI, D.M.S.S.; ABDALLA, A.L.; MEIRELES, C.S. Cinética de fósforo em ovinos suplementados com diferentes fontes fosfatadas, através da técnica de diluição isotópica. Arquivo Brasileiro de Medicina Veterinária e Zootecnia, Belo Horizonte, v.44, n.3, p.227-233, 1992a.

VITTI, D.M.S.S.; ABDALLA, A.L.; MEIRELES, C.S.; SILVA FILHO, J.C. Avaliação da disponibilidade biológica do fósforo do fosfato bicálcico e de fosfatos de rocha para ovinos com uso do radiofósforo $\left({ }^{32} \mathrm{P}\right)$ como traçador. Pesquisa Agropecuária Brasileira, Brasília, v.26, n.8, p.1113-1118, ago. 1991.

VITTI, D.M.S.S.; ABDALLA, A.L.; MEIRELES, C.S.; SILVA FILHO, J.C.; LOUVANDINI, H. Absorção real do fósforo de diferentes fontes para ovinos através do uso de radiofósforo (P-32). Pesquisa Agropecuária Brasileira, Brasília, v.27, n.10, p.1405-1408, out. 1992 b.

VITTI, D.M.S.S.; ABDALLA, A.L.; SILVA FILHO, J.C. Fontes alternativas de fósforo para ruminantes: $a b-$ sorção real e disponibilidade biológica. Arquivo Brasileiro de Medicina Veterinária e Zootecnia, Belo Horizonte, v.41, n.6, p.503-510, 1989. 